\title{
Enhancement of Loading of Radial Distribution Networks Using Optimal Conductor Size
}

\author{
Smarajit Ghosh, Uttamjit Singh Chhatwal
}

\begin{abstract}
In this paper, an attempt has been made to enhance the loading of radial distribution by selection of proper conductor. The critical values of total real power load (TPL) and total reactive power load (TQL) for constant power are derived out for the sub-station voltage of $1.0 \mathrm{pu}$. for 69-node radial distribution network. The proposed method will reduce the real and reactive power losses, improves voltage profile and enhances the loading capability of distribution network. The voltage deviation is reduced to $\pm 10 \%$. The superiority of the proposed method has been established by $\pm 6 \%$ from $\pm 10 \%$ taking results iteratively. Using network reconfiguration, loading is further increased.
\end{abstract}

Index Terms-Load-flow, Optimal, Conductor, Network, Reconfiguration, Power load

\section{INTRODUCTION}

A fast algorithm to help the distribution engineer to select proper size of conductors for one's future expansion plan is utmost important because it increases the loading of the system. The optimal conductor type is determined for each feeder segment to maintain an acceptable voltage profile of the entire feeder, minimizing capital investment and the cost of feeder losses. An optimal model for configuring feeder networks to derive an overall expansion was discussed by Tram and Wall [1]. Although their algorithm was based on realistic assumptions and reliable optimization techniques, it was relatively complicated to handle for the engineer. A model to represent feeder cost, energy loss cost and voltage regulation as a function of conductor cross-section and an objective function for optimizing the conductor cross-section had been formulated by Ponnavaikkio and Prakasa Rao [2]. The method proposed in [2] had taken into account the non-uniform distribution of loads along with the length of feeder and load growth in the future years of the plan period. A direct solution procedure for conductor grading was proposed, thereby eliminating the complexity of the dynamic programming approach by Rao [3] A new computational procedure for obtaining the optimal conductor grading policy using PPR model was proposed, which was extremely simple and require very little computation and needed very little computer storage. Methods [1] and [3] could solve problems accurately for small scale whereas these two methods could not guarantee for optimal solution for large scale problem.

In [4] a new technique of optimizing the feeder routing problem which minimized the total cost of feeder subject to a

Smarajit Ghosh is with the Thapar University, Department of Electrical \& Instrumentation Engineering, Patiala, Punjab-147004, INDIA

Uttamjit Singh Chhatwal is with BILT(Thapar Group), Yamunanagar, Haryana, INDIA set of specified constraints was discussed. Ponnavaikko and Rao [2] developed a mathematical model for the problem, and presented multi stage decision dynamic programming method. However, this method failed in handling the large problem and showed only approximate results. The approach discussed by Wang et al. [5] included an economical current density based method and a heuristic method, which together enabled a satisfactory solution that could be easily achieved. Miu and Chiang [6] proposed a solution algorithm to determine distribution loading capability. A solution algorithm suitable for large-scale unbalanced distribution networks with capacitor control actions was developed and tested. However, their model was suitable only for constant current load and for radial main feeder only.

Das [7] presented a simple algorithm for determining the maximum loading of the feeders without violating the maximum current capacity of branch conductor. A predetermined annual load growth was also considered to determine allowable load growth period without violating the minimum voltage limit of the feeder. A dynamic model for the development of primary and secondary circuits supplying a residential area had been proposed by Kirn and Adler [8]. Features of the model which support optimal conductor sizing were the evaluation of annual revenue requirements associated with capital requirement and energy losses as area load evolves. These revenue requirements were responsive to change in area load (positive or negative) arising with change in the number of residences and change in the load per residence year by year. Results of optimization trials explored the relative penalties incurred for optimal conductor policies based on incorrect projections of load growth, degree of load management expected, and costs of losses.

A method was proposed for selecting the optimal size of branch conductor for radial distribution network by Satyanarayana et al. [9]. In the proposed method [9], the conductor would not only maintain maximum current carrying capacity but also maintain the voltage level for the distribution network.

Two algorithms for the reconfiguration of feeders was presented by Ghosh and Das [10] with two heuristic rules. In the first heuristic rule closing of an open tie switch was based on the maximum voltage drop across it during iterative process and this drop should be greater than some specified value. In the first heuristic rule closing of an open switch is arbitrary, but the voltage drop across it must be greater than some specified value. The load-flow proposed by S. Ghosh et al.[10] is used in the present paper.

The aim of authors is to propose a method to pick up the optimal conductor to enhance the loading of the system. Further, the network reconfiguration [10] is applied to 
enhance the loading. 69-node radial distribution network [12] is selected to demonstrate this method.

\section{ASSUMPTION}

The three phase radial distribution networks are assumed to be balanced and can be represented by their single line diagram. Load is assumed to be constant power load.

\section{NETWORK RECONFIGURATION}

Radial distribution networks have some advantages over mesh distribution networks such as lower short circuit current and simpler switching and protecting equipment. On the other hand, the radial structure provides lower overall reliability. Therefore, to use the benefits of the radial structure and at the same time to overcome the difficulties, distribution systems are planned and built as weakly meshed networks, but operated as radial networks.

Distribution systems consist of group of interconnected radial circuits. Their configuration may varied with manual or automatic switching operations to transfer loads among the feeders. There are two kind of switching in the primary distribution systems, namely, normally closed switches that connect line sections and normally open switches on the tie lines that connect two primary feeders, two substations, or loop type laterals. The former is called sectionalizing switches and the latter is called tie-lines switch. Both of them are designed for fault isolation and network reconfiguration. The purpose of network reconfiguration is to produce the minimum loss possible under the circuit's capacity constraint.

\section{SOLUTION METHODOLOGY}

69-node radial distribution network [12] is taken as example. Initially the network has 5 tie-lines switches and connecting the nodes $(11,43),(13,21),(15,46),(27,65),(50$, 59). At first load-flow is run to compute the branch currents and node voltages. The results of load-flow will give branch current using these branch currents conductor as per their maximum current carrying capability is being selected for their respective branch. In the present paper work, conductor is loaded $85 \%$ of its maximum value. The reason for not taking $100 \%$ loading of conductor is that during fault condition if conductor is $100 \%$ loaded then it will be burnt out.

Load-flow also gives voltages at every node, the percentage deviation of voltage w.r.t to p.u. voltage is calculated and it is derived that one has to operate on those nodes having minimum percentage voltage deviation. Then according to percentage voltage deviation the nodes are arranged in ascending order. The nodes having percentage deviation less than 1 are selected. According to voltage deviation the load is distributed on the branches.

Fig. 1 shows a sample radial distribution network. Table I shows the branch number, sending-end node, receiving-end node of Fig. 1.

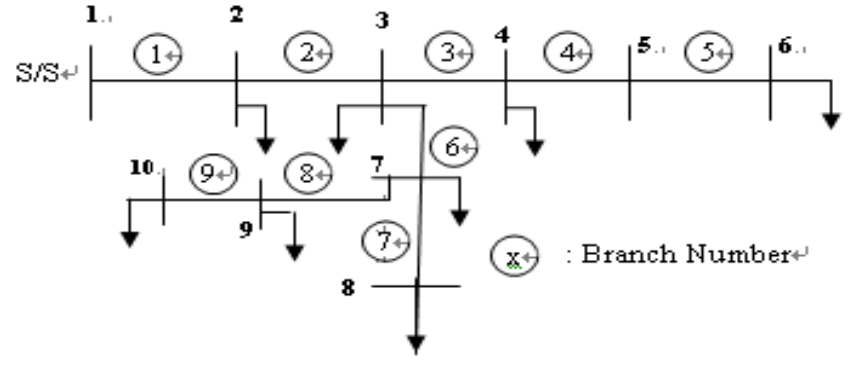

Fig. 1 Single-line diagram of a radial distribution network

TABLE 1 BRANCH NUMBER (JJ), SENDING END NODE $(\mathrm{M} 1=\mathrm{IS}(\mathrm{JJ}))$, RECEIVING END NODE $(\mathrm{M} 2=\mathrm{IR}(\mathrm{JJ}))$ AND NODES BEYOND BRANCHES $1,2,3, \ldots, 10$ OF FIG. 1

\begin{tabular}{|c|c|c|}
\hline $\begin{array}{c}\text { Branch } \\
\text { Number } \\
\text { jj) }\end{array}$ & $\begin{array}{c}\text { Sending-end } \\
\text { nodes }\end{array}$ & $\begin{array}{c}\text { Receiving-end } \\
\text { nodes }\end{array}$ \\
\hline 1 & 2 & $\mathbf{2}$ \\
\hline 2 & 3 & 4 \\
\hline 3 & 4 & 5 \\
\hline 4 & 5 & 6 \\
\hline 5 & 3 & 7 \\
\hline 6 & 7 & 8 \\
\hline 7 & 7 & 10 \\
\hline 8 & 9 & 9 \\
\hline 9 & 2 & 5 \\
\hline
\end{tabular}

Table II shows the nodes beyond each branch of Fig.1.

TABLE II NODES BEYOND BRANCHES $1,2,3, \ldots, 10$ OF FIG. 1

\begin{tabular}{|c|c|}
\hline $\begin{array}{c}\text { Branch } \\
\text { Number }(\mathbf{j j})\end{array}$ & Nodes beyond each branch (jj) \\
\hline 1 & $2,3,4,5,6,7,8,9,10$ \\
\hline 2 & $3,4,5,6,7,8,9,10$ \\
\hline 3 & $4,5,6$ \\
\hline 4 & 5,6 \\
\hline 5 & 6 \\
\hline 6 & $7,8,9,10$ \\
\hline 7 & 8 \\
\hline 8 & 10 \\
\hline
\end{tabular}

The voltage at any receiving-end node $(\mathrm{m} 2)$ of branch-jj is expressed by

$$
\begin{gathered}
V(m 2)=V(m 1)-I(j j) Z(j j) \\
\text { i.e., } V(m 2)=V(m 1)-I(j j)[R(j j)+j X(j j)] \\
\text { where, } m 1=I S(j j) \\
\text { and } m 2=I R(j j)
\end{gathered}
$$

The load current of any receiving-end node $\mathrm{m} 2=\operatorname{IR}(\mathrm{jj})$ of branch-jj is expressed by 


$$
\mathrm{IL}(\mathrm{m} 2)=\frac{P L(\mathrm{~m} 2)-\mathrm{jQL}(\mathrm{m} 2)}{\mathrm{V}^{*}(\mathrm{~m} 2)}
$$

The real and reactive power losses of branch-jj are expressed by

$$
\begin{gathered}
L P=|I(j j)|^{2} R(j j) \\
\text { and } L Q=|I(j j)|^{2} X(j j)
\end{gathered}
$$

respectively.

The current through branch-jj is the sum of all load currents of all nodes beyond branch-jj i.e.,

$$
I(j j)=\sum_{i=1}^{N(j i)} \operatorname{IL}\{I E(j j, i)\}
$$

where $\mathrm{N}(\mathrm{jj})$ is the total number of nodes beyond branch $\mathrm{jj}$ and $\mathrm{IE}(\mathrm{jj}, \mathrm{i})$ is the receiving-end node beyond branch-jj. The algorithm for identification of nodes beyond each branch-jj is available in [11]. Table II shows the nodes beyond each branch of Fig. 1. Let $\operatorname{CImax}(\mathrm{jj})$ be the maximum current capacity of the conductor of branch-jj.After running the load-flow, current though the each branch and voltage of each node can be obtained. The difference $\Delta \mathbf{I}(\mathbf{j} \mathbf{j})=$ $\mathbf{0 . 8 5} \times \mathbf{C I m a x}(\mathbf{j} \mathbf{j})-\mathbf{I}(\mathbf{j} \mathbf{j})$ can be computed. The percentage change in voltage of each node is arranged in ascending order. The node having the voltage less than $1.0000 \mathrm{pu}$ are taken into account only. The load of this node is increased slowly

\begin{tabular}{|c|c|c|}
\hline Step -1 & $:$ & Start \\
\hline Step -2 & : & $\begin{array}{l}\text { Read sending-end and } \\
\text { receiving-end nodes and total } \\
\text { number of nodes and branches }\end{array}$ \\
\hline Step -3 & : & $\begin{array}{l}\text { Set } V(i)=1.0+j 0.0 \text { for all } \mathrm{i}= \\
1,2,3, \ldots, N B\end{array}$ \\
\hline Step -4 & : & $\begin{array}{l}\text { Set ISS }(j j)=\operatorname{IS}(j j) \text { and } \operatorname{IRR}(j \mathrm{j}) \\
=\operatorname{IR}(\mathrm{jj}) \text { for } \mathrm{jj}=1,2,3, \ldots \ldots \text {, } \\
\mathrm{LN} 1\end{array}$ \\
\hline Step -5 & $:$ & Set iteration count $\mathrm{k}=1$. \\
\hline Step -6 & $:$ & Set kMAX = 100 (say) \\
\hline Step -7 & $:$ & $\begin{array}{l}\text { Set DVMAX }=0.0 \text { and } \varepsilon= \\
0.00001\end{array}$ \\
\hline Step -8 & : & $\begin{array}{l}\text { Identify the nodes beyond } \\
\text { each branch using IDENT } \\
\text { software [11]. }\end{array}$ \\
\hline Step -9 & $:$ & $\begin{array}{l}\text { Compute load currents } \\
\mathrm{IL}(\mathrm{m} 2) \text { for } \mathrm{m} 2=1,2,3, \ldots, \\
\text { NB using Eq.[5] }\end{array}$ \\
\hline Step -10 & $:$ & $\begin{array}{l}\text { Compute current through each } \\
\text { branch i.e., I(jj) for } \mathrm{jj}=1,2 \text {, } \\
3, \ldots, \mathrm{LN} 1 \text { using Eq.[8] }\end{array}$ \\
\hline Step -11 & $:$ & Set $\mathrm{jj}=1$ \\
\hline Step -12 & $:$ & $\begin{array}{l}\text { Compute } \quad \Delta \mathrm{I}(\mathrm{jj}) \\
0.85 \times \operatorname{CImax}(\mathrm{jj})-\mathrm{I}(\mathrm{jj})\end{array}$ \\
\hline Step - 13 & : & $\begin{array}{l}\text { Set } m 1=\operatorname{ISS}(\mathrm{jj}) \text { and } \mathrm{m} 2= \\
\operatorname{IRR}(\mathrm{jj}) . \\
\text { Compute }\end{array}$ \\
\hline
\end{tabular}
until the $\Delta \mathbf{I}(\mathbf{j} \mathbf{j})$ remains positive. The network is reconfigured and the similar technique is applied. The complete algorithm is shown below.

\begin{tabular}{|c|c|c|}
\hline Step - 14 & $:$ & $\begin{array}{l}\text { Compute the voltage } \\
\text { deviation } \mathrm{VD}(\mathrm{m} 2)=1-\mathrm{V}(\mathrm{m} 2)\end{array}$ \\
\hline Step -15 & $:$ & $\mathrm{jj}=\mathrm{jj}+1$ \\
\hline Step -16 & $:$ & $\begin{array}{l}\text { If } \mathrm{jj}<\mathrm{LN} 1 \text {, go to Step-13, } \\
\text { otherwise go to next Step-17. }\end{array}$ \\
\hline Step -17 & $:$ & $\begin{array}{l}\text { Find the percentage voltage } \\
\text { deviation } \operatorname{VD}(\mathrm{m} 2)\end{array}$ \\
\hline Step -18 & : & $\begin{array}{l}\text { Arrange percentage voltage } \\
\text { deviation } \operatorname{VD}(\mathrm{m} 2) \text { in } \\
\text { ascending order. }\end{array}$ \\
\hline Step - 19 & $:$ & $\begin{array}{l}\text { If all } \mathrm{VD}(\mathrm{m} 2)>1.000 \text {, go to } \\
\text { Step-25 }\end{array}$ \\
\hline Step -20 & $:$ & Set $\mathrm{m} 2=0$ \\
\hline Step -21 & $:$ & $\begin{array}{l}\text { Calculated total number of } \\
\text { nodes having } \operatorname{VD}(\mathrm{m} 2)< \\
1.000 \text {. }\end{array}$ \\
\hline Step -22 & $:$ & $\mathrm{m} 3=\mathrm{m} 2+1$ \\
\hline Step -23 & $:$ & $\begin{array}{l}\text { Increase pl \& } \mathrm{ql} \text { till } \Delta \mathrm{I}(\mathrm{jj}) \text { is } \\
\text { positive. }\end{array}$ \\
\hline Step -24 & $:$ & Print "Results" \\
\hline Step -25 & $:$ & Stop \\
\hline
\end{tabular}

receiving-end voltage $\mathrm{V}(\mathrm{m} 2)$ for all $\mathrm{m} 2$ using Eq. [2].

\section{EXAMPLE}

A 69-node radial distribution network is shown in Fig. 2. Base values are $12.66 \mathrm{kV}$ and $100 \mathrm{MVA}$. Load data and line data for 69-node radial distribution network are available in [12]. Initially the real and reactive power losses of radial network are $224.933 \mathrm{~kW}$ and $102.129 \mathrm{kVAr}$ respectively. Node 65 has the lowest voltage level i.e $0.9091 \mathrm{pu}$. The total real and reactive power load on the system are $3801.89 \mathrm{~kW}$ and $2692.60 \mathrm{kVAr}$ respectively. Table III shows the branch current, maximum current rating and allowable current rating and their difference for 69-node radial distribution network. Fig. 3 shows the 69-node radial distribution network after network reconfiguration using the method of [12]. Table IV shows branch current of 69-node radial distribution network [12] after network reconfiguration. Table V Branch Current of 69-node Radial Distribution Network after network reconfiguration. Table VI percentage voltage deviation w.r.t to p.u. voltage of 69-node radial distribution network after network reconfiguration in ascending order. Table VII percentage voltage deviation w.r.t pu voltage of 69-node radial distribution network [12] in ascending order for percentage deviation less than 1.000 after network reconfiguration. Table VIII shows increase in real power (PL) and reactive power (QL) load of 69 node radial distribution network [10] after network reconfiguration. Table IX shows comparison of real and reactive power losses. Table X shows comparison of minimum pu voltage level and respective node number. Table XI shows comparison of total real and reactive power load. 


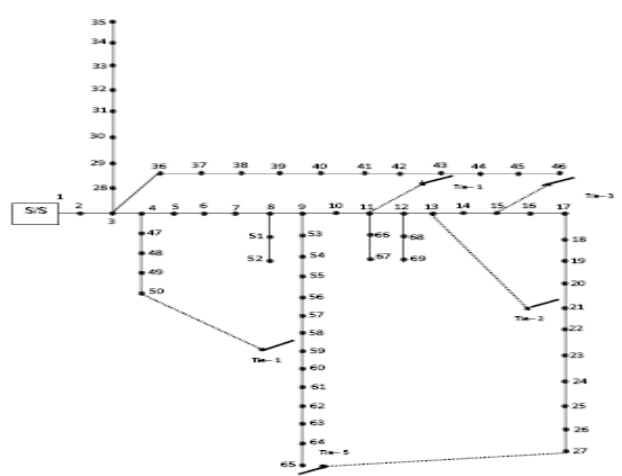

Fig. 269 node radial distribution network [12]

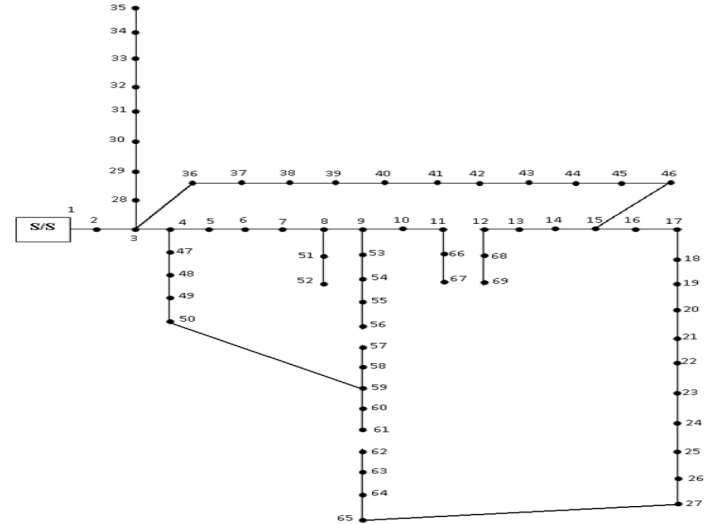

Fig. 3 Reconfigured 69 Node Radial Distribution Network [10]

TABLE III BRANCH CURRENT, MAXIMUM CURRENT RATING AND ALLOWABLE CURRENT RATING AND THEIR DIFFERENCE FOR 69-NODE RADIAL DISTRIBUTION NETWORK [12]

\begin{tabular}{|c|c|c|c|c|c|}
\hline Branch Number & Branch Current & Conductor Name & $\begin{array}{l}\text { Maximum Current } \\
\text { Rating }\end{array}$ & $\begin{array}{c}\text { Allowable Current } \\
\text { Rating }\end{array}$ & Difference \\
\hline 1 & 223.53 & CAT & 290 & 246.5 & 22.96 \\
\hline 2 & 223.53 & CAT & 290 & 246.5 & 22.97 \\
\hline 3 & 208.09 & CAT & 290 & 246.5 & 38.41 \\
\hline 4 & 160.35 & FERRET & 175 & 148.75 & 9.65 \\
\hline 5 & 160.35 & FERRET & 175 & 148.75 & 9.65 \\
\hline 6 & 160.19 & FERRET & 175 & 148.75 & 9.81 \\
\hline 7 & 157.85 & FERRET & 175 & 148.75 & 12.15 \\
\hline 8 & 151.03 & FERRET & 175 & 148.75 & 18.97 \\
\hline 9 & 44.06 & MOLE & 70 & 59.5 & 15.44 \\
\hline 10 & 42.47 & MOLE & 70 & 59.5 & 17.03 \\
\hline 11 & 32.01 & MOLE & 70 & 59.5 & 27.49 \\
\hline 12 & 20.37 & MOLE & 70 & 59.5 & 39.13 \\
\hline 13 & 19.93 & MOLE & 70 & 59.5 & 39.57 \\
\hline 14 & 19.48 & MOLE & 70 & 59.5 & 40.02 \\
\hline 15 & 19.48 & MOLE & 70 & 59.5 & 40.02 \\
\hline 16 & 16.89 & MOLE & 70 & 59.5 & 42.61 \\
\hline 17 & 13.59 & MOLE & 70 & 59.5 & 45.91 \\
\hline 18 & 10.29 & MOLE & 70 & 59.5 & 49.21 \\
\hline 19 & 10.29 & MOLE & 70 & 59.5 & 49.21 \\
\hline 20 & 10.24 & MOLE & 70 & 59.5 & 49.26 \\
\hline 21 & 3.57 & SQUIRREL & 120 & 102 & 55.93 \\
\hline 22 & 3.28 & SQUIRREL & 120 & 102 & 56.22 \\
\hline 23 & 3.28 & SQUIRREL & 120 & 102 & 56.22 \\
\hline 24 & 1.64 & SQUIRREL & 120 & 102 & 57.86 \\
\hline 25 & 1.64 & SQUIRREL & 120 & 102 & 57.86 \\
\hline 26 & 0.82 & SQUIRREL & 120 & 102 & 8.68 \\
\hline 27 & 5.13 & SQUIRREL & 120 & 102 & 4.37 \\
\hline 28 & 3.67 & SQUIRREL & 120 & 102 & 55.83 \\
\hline 29 & 2.21 & SQUIRREL & 120 & 102 & 57.29 \\
\hline 30 & 2.21 & SQUIRREL & 120 & 102 & 57.29 \\
\hline 31 & 2.21 & SQUIRREL & 120 & 102 & 57.29 \\
\hline 32 & 2.21 & SQUIRREL & 120 & 102 & 57.29 \\
\hline 33 & 1.42 & MOLE & 70 & 59.5 & 58.08 \\
\hline 34 & 0.33 & MOLE & 70 & 59.5 & 59.17 \\
\hline 35 & 10.32 & MOLE & 70 & 59.5 & 49.18 \\
\hline 36 & 8.86 & MOLE & 70 & 59.5 & 50.64 \\
\hline 37 & 7.41 & MOLE & 70 & 59.5 & 52.09 \\
\hline 38 & 7.41 & MOLE & 70 & 59.5 & 52.09 \\
\hline 39 & 6.06 & MOLE & 70 & 59.5 & 53.44 \\
\hline 40 & 4.72 & MOLE & 70 & 59.5 & 54.78 \\
\hline 41 & 4.65 & MOLE & 70 & 59.5 & 54.85 \\
\hline 42 & 4.65 & MOLE & 70 & 59.5 & 54.85 \\
\hline 43 & 4.31 & MOLE & 70 & 59.5 & 55.19 \\
\hline 44 & 4.31 & MOLE & 70 & 59.5 & 55.19 \\
\hline 45 & 2.16 & MOLE & 70 & 59.5 & 57.34 \\
\hline 46 & 47.75 & MOLE & 70 & 59.5 & 11.75 \\
\hline 47 & 47.75 & MOLE & 70 & 59.5 & 11.75 \\
\hline 48 & 43.32 & MOLE & 70 & 59.5 & 16.18 \\
\hline 49 & 21.67 & MOLE & 70 & 59.5 & 37.83 \\
\hline 50 & 2.51 & MOLE & 70 & 59.5 & 56.99 \\
\hline 51 & 0.21 & MOLE & 70 & 59.5 & 59.29 \\
\hline
\end{tabular}




\begin{tabular}{|c|c|c|c|c|c|}
\hline 52 & 105.24 & MOLE & 70 & 59.5 & 5.26 \\
\hline 53 & 104.98 & MOLE & 70 & 59.5 & 5.52 \\
\hline 54 & 103.45 & SQUIRREL & 120 & 102 & 7.05 \\
\hline 55 & 102.06 & SQUIRREL & 120 & 102 & 8.44 \\
\hline 56 & 102.06 & SQUIRREL & 120 & 102 & 8.44 \\
\hline 57 & 102.06 & SQUIRREL & 120 & 102 & 8.44 \\
\hline 58 & 102.06 & SQUIRREL & 120 & 102 & 8.44 \\
\hline 59 & 95.98 & GOPHER & 130 & 110.5 & 14.52 \\
\hline 60 & 95.98 & GOPHER & 130 & 110.5 & 14.52 \\
\hline 61 & 19.58 & MOLE & 70 & 59.5 & 39.92 \\
\hline 62 & 17.61 & SQUIRREL & 70 & 59.5 & 41.89 \\
\hline 63 & 17.61 & MOLE & 70 & 59.5 & 41.89 \\
\hline 64 & 3.63 & MOLE & 70 & 59.5 & 55.87 \\
\hline 65 & 2.09 & MOLE & 70 & 59.5 & 57.41 \\
\hline 66 & 1.04 & MOLE & 70 & 59.5 & 58.46 \\
\hline 67 & 3.24 & MOLE & 70 & 59.5 & 56.26 \\
\hline 68 & 1.62 & MOLE & 70 & 59.5 & 57.88 \\
\hline
\end{tabular}

TABLE IV BRANCH CURRENT, MAXIMUM CURRENT RATING AND ALLOWABLE CURRENT RATING AND THEIR DIFFERENCE FOR 69-NODE RADIAL DISTRIBUTION NETWORK AFTER NETWORK RECONFIGURATION

\begin{tabular}{|c|c|c|c|c|c|}
\hline Branch Number & Branch Current & Conductor Name & $\begin{array}{c}\text { Maximum Current } \\
\text { Rating } \\
\end{array}$ & $\begin{array}{c}\text { Allowable Current } \\
\text { Rating } \\
\end{array}$ & Difference \\
\hline 1 & 219.0057 & CAT & 290 & 246.5 & 27.4943 \\
\hline 2 & 219.0057 & CAT & 290 & 246.5 & 27.4943 \\
\hline 3 & 153.1999 & CAT & 290 & 246.5 & 93.3001 \\
\hline 4 & 25.7184 & MOLE & 70 & 59.5 & 33.7816 \\
\hline 5 & 25.7184 & MOLE & 70 & 59.5 & 33.7816 \\
\hline 6 & 25.5633 & MOLE & 70 & 59.5 & 33.9367 \\
\hline 7 & 23.2614 & MOLE & 70 & 59.5 & 36.2386 \\
\hline 8 & 16.5651 & MOLE & 70 & 59.5 & 42.9349 \\
\hline 9 & 11.7665 & MOLE & 70 & 59.5 & 47.7335 \\
\hline 10 & 10.2162 & MOLE & 70 & 59.5 & 49.2838 \\
\hline 11 & 2.0359 & MOLE & 70 & 59.5 & 57.4641 \\
\hline 12 & 1.0179 & MOLE & 70 & 59.5 & 58.4821 \\
\hline 13 & 5.1261 & MOLE & 70 & 59.5 & 54.3739 \\
\hline 14 & 3.6681 & MOLE & 70 & 59.5 & 55.8319 \\
\hline 15 & 2.2100 & MOLE & 70 & 59.5 & 57.2900 \\
\hline 16 & 2.2100 & MOLE & 70 & 59.5 & 57.2900 \\
\hline 17 & 2.2100 & MOLE & 70 & 59.5 & 57.2900 \\
\hline 18 & 2.2100 & MOLE & 70 & 59.5 & 57.2900 \\
\hline 19 & 1.4249 & MOLE & 70 & 59.5 & 58.0751 \\
\hline 20 & 0.3292 & MOLE & 70 & 59.5 & 59.1708 \\
\hline 21 & 60.6870 & SQUIRREL & 120 & 102 & 41.3130 \\
\hline 22 & 59.2304 & MOLE & 70 & 59.5 & 0.2696 \\
\hline 23 & 57.7721 & MOLE & 70 & 59.5 & 1.7279 \\
\hline 24 & 57.7721 & MOLE & 70 & 59.5 & 1.7279 \\
\hline 25 & 56.4270 & MOLE & 70 & 59.5 & 3.0730 \\
\hline 26 & 55.0819 & MOLE & 70 & 59.5 & 4.4181 \\
\hline 27 & 55.0102 & MOLE & 70 & 59.5 & 4.4898 \\
\hline 28 & 55.0102 & MOLE & 70 & 59.5 & 4.4898 \\
\hline 29 & 54.6685 & MOLE & 70 & 59.5 & 4.8315 \\
\hline 30 & 54.6685 & MOLE & 70 & 59.5 & 4.8315 \\
\hline 31 & 52.4793 & MOLE & 70 & 59.5 & 7.0207 \\
\hline 32 & 50.2901 & MOLE & 70 & 59.5 & 9.2099 \\
\hline 33 & 37.7662 & MOLE & 70 & 59.5 & 21.7338 \\
\hline 34 & 35.2174 & MOLE & 70 & 59.5 & 24.2826 \\
\hline 35 & 31.9716 & MOLE & 70 & 59.5 & 27.5284 \\
\hline 36 & 28.7278 & MOLE & 70 & 59.5 & 30.7722 \\
\hline 37 & 28.7278 & MOLE & 70 & 59.5 & 30.7722 \\
\hline 38 & 28.6732 & MOLE & 70 & 59.5 & 30.8268 \\
\hline 39 & 22.0961 & MOLE & 70 & 59.5 & 37.4039 \\
\hline 40 & 21.8090 & MOLE & 70 & 59.5 & 37.6910 \\
\hline 41 & 21.8090 & MOLE & 70 & 59.5 & 37.6910 \\
\hline 42 & 20.1881 & MOLE & 70 & 59.5 & 39.3119 \\
\hline 43 & 20.1881 & MOLE & 70 & 59.5 & 39.3119 \\
\hline 44 & 19.3752 & MOLE & 70 & 59.5 & 40.1248 \\
\hline 45 & 18.5619 & MOLE & 70 & 59.5 & 40.9381 \\
\hline 46 & 15.1283 & MOLE & 70 & 59.5 & 44.3717 \\
\hline 47 & 1.8735 & MOLE & 70 & 59.5 & 57.6265 \\
\hline 48 & 1.8735 & MOLE & 70 & 59.5 & 57.6265 \\
\hline 49 & 12.5258 & MOLE & 70 & 59.5 & 46.9742 \\
\hline 50 & 12.0714 & MOLE & 70 & 59.5 & 47.4286 \\
\hline 51 & 11.6162 & MOLE & 70 & 59.5 & 47.8838 \\
\hline
\end{tabular}




\begin{tabular}{|c|c|c|c|c|c|}
\hline 52 & 3.2339 & MOLE & 70 & 59.5 & 56.2661 \\
\hline 53 & 1.6169 & MOLE & 70 & 59.5 & 57.8831 \\
\hline 54 & 127.4816 & FERRET & 175 & 148.75 & 21.2684 \\
\hline 55 & 127.4816 & FERRET & 175 & 148.75 & 21.2684 \\
\hline 56 & 123.0390 & FERRET & 175 & 148.75 & 25.7110 \\
\hline 57 & 101.1829 & GOPHER & 130 & 110.5 & 9.3171 \\
\hline 58 & 79.2705 & SQUIRREL & 120 & 102 & 22.7295 \\
\hline 59 & 110.5000 & GOPHER & 130 & 110.5 & 0.9590 \\
\hline 60 & 110.5000 & GOPHER & 130 & 110.5 & 0.9590 \\
\hline 61 & 73.4108 & SQUIRREL & 120 & 102 & 28.5892 \\
\hline 62 & 73.4108 & SQUIRREL & 120 & 102 & 28.5892 \\
\hline 63 & 2.4670 & MOLE & 70 & 59.5 & 57.0330 \\
\hline 64 & 0.2059 & MOLE & 70 & 59.5 & 59.2941 \\
\hline 65 & 3.0960 & MOLE & 70 & 59.5 & 56.4040 \\
\hline 66 & 2.8407 & MOLE & 70 & 59.5 & 56.6593 \\
\hline 67 & 1.3518 & MOLE & 70 & 59.5 & 58.1482 \\
\hline 68 & 59.5000 & MOLE & 70 & 59.5 & 0.9961 \\
\hline
\end{tabular}

TABLE V BRANCH CURRENT OF 69-NODE RADIAL DISTRIBUTION NETWORK AFTER NETWORK RECONFIGURATION.

\begin{tabular}{|c|c|c|c|c|c|}
\hline Branch Number & Branch Current & Conductor Name & $\begin{array}{l}\text { Maximum Current } \\
\text { Rating } \\
\end{array}$ & $\begin{array}{c}\text { Allowable Current } \\
\text { Rating } \\
\end{array}$ & Difference \\
\hline 1 & 219.006 & CAT & 290 & 246.5 & 27.494 \\
\hline 2 & 219.006 & CAT & 290 & 246.5 & 27.494 \\
\hline 4 & 25.718 & MOLE & 70 & 59.5 & 33.782 \\
\hline 5 & 25.718 & MOLE & 70 & 59.5 & 33.782 \\
\hline 6 & 25.563 & MOLE & 70 & 59.5 & 33.937 \\
\hline 8 & 16.565 & MOLE & 70 & 59.5 & 42.935 \\
\hline 9 & 11.767 & MOLE & 70 & 59.5 & 47.733 \\
\hline 10 & 10.216 & MOLE & 70 & 59.5 & 49.284 \\
\hline 11 & 2.036 & MOLE & 70 & 59.5 & 57.464 \\
\hline 12 & 1.018 & MOLE & 70 & 59.5 & 58.482 \\
\hline 13 & 5.126 & MOLE & 70 & 59.5 & 54.374 \\
\hline 14 & 3.668 & MOLE & 70 & 59.5 & 55.832 \\
\hline 15 & 2.210 & MOLE & 70 & 59.5 & 57.290 \\
\hline 17 & 2.210 & MOLE & 70 & 59.5 & 57.290 \\
\hline 18 & 2.210 & MOLE & 70 & 59.5 & 57.290 \\
\hline 19 & 1.425 & MOLE & 70 & 59.5 & 58.075 \\
\hline 20 & 0.329 & MOLE & 70 & 59.5 & 59.171 \\
\hline 21 & 60.687 & SQUIRREL & 120 & 102 & 41.313 \\
\hline 22 & 59.230 & MOLE & 70 & 59.5 & 0.270 \\
\hline 23 & 57.772 & MOLE & 70 & 59.5 & 1.728 \\
\hline 24 & 57.772 & MOLE & 70 & 59.5 & 1.728 \\
\hline 25 & 56.427 & MOLE & 70 & 59.5 & 3.073 \\
\hline 26 & 55.082 & MOLE & 70 & 59.5 & 4.418 \\
\hline 27 & 55.010 & MOLE & 70 & 59.5 & 4.490 \\
\hline 28 & 55.010 & MOLE & 70 & 59.5 & 4.490 \\
\hline 29 & 54.668 & MOLE & 70 & 59.5 & 4.832 \\
\hline 30 & 54.668 & MOLE & 70 & 59.5 & 4.832 \\
\hline 31 & 52.479 & MOLE & 70 & 59.5 & 7.021 \\
\hline 34 & 35.217 & MOLE & 70 & 59.5 & 24.283 \\
\hline 35 & 31.972 & MOLE & 70 & 59.5 & 27.528 \\
\hline 36 & 28.728 & MOLE & 70 & 59.5 & 30.772 \\
\hline 37 & 28.728 & MOLE & 70 & 59.5 & 30.772 \\
\hline 38 & 28.673 & MOLE & 70 & 59.5 & 30.827 \\
\hline 39 & 22.096 & MOLE & 70 & 59.5 & 37.404 \\
\hline 40 & 21.809 & MOLE & 70 & 59.5 & 37.691 \\
\hline 41 & 21.809 & MOLE & 70 & 59.5 & 37.691 \\
\hline 42 & 20.188 & MOLE & 70 & 59.5 & 39.312 \\
\hline 43 & 20.188 & MOLE & 70 & 59.5 & 39.312 \\
\hline 44 & 19.375 & MOLE & 70 & 59.5 & 40.125 \\
\hline 45 & 18.562 & MOLE & 70 & 59.5 & 40.938 \\
\hline 46 & 15.128 & MOLE & 70 & 59.5 & 44.372 \\
\hline 47 & 1.874 & MOLE & 70 & 59.5 & 57.626 \\
\hline 48 & 1.874 & MOLE & 70 & 59.5 & 57.626 \\
\hline 49 & 12.526 & MOLE & 70 & 59.5 & 46.974 \\
\hline 50 & 12.071 & MOLE & 70 & 59.5 & 47.429 \\
\hline 51 & 11.616 & MOLE & 70 & 59.5 & 47.884 \\
\hline
\end{tabular}




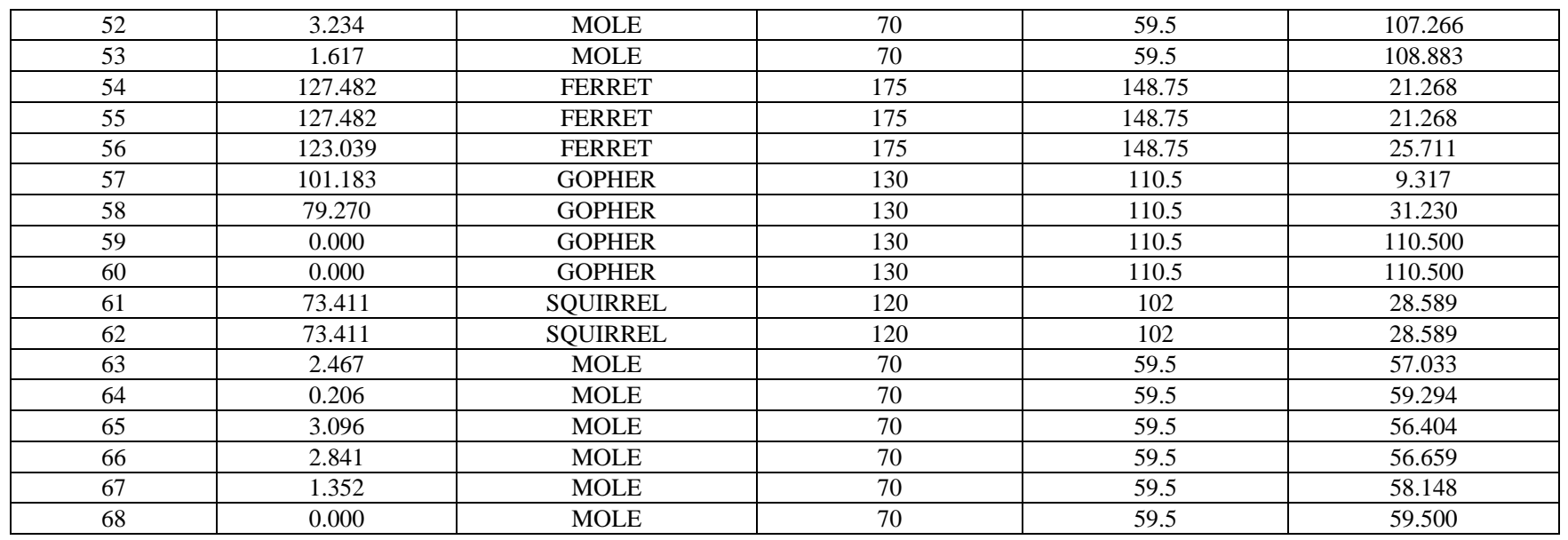

TABLE VI \% VOLTAGE DEVIATION W.R.T TO PU VOLTAGE OF 69-NODE RADIAL DISTRIBUTION NETWORK AFTER NETWORK RECONFIGURATION IN ASCENDING ORDER

\begin{tabular}{|c|c|c|c|c|c|}
\hline Node Number & \% Voltage Deviation & Node Number & \%Voltage Deviation & Node Number & $\%$ Voltage Deviation \\
\hline 2 & 0.003696 & 55 & 0.879171 & 12 & 4.426449 \\
\hline 3 & 0.007392 & 56 & 0.886056 & 68 & 4.458965 \\
\hline 28 & 0.009448 & 49 & 0.956970 & 69 & 4.459141 \\
\hline 4 & 0.013848 & 10 & 1.118716 & 20 & 4.536425 \\
\hline 29 & 0.019095 & 50 & 1.163702 & 21 & 4.744587 \\
\hline 36 & 0.022452 & 11 & 1.176740 & 22 & 4.752645 \\
\hline 30 & 0.038472 & 66 & 1.200969 & 23 & 4.838912 \\
\hline 5 & 0.044765 & 67 & 1.200999 & 57 & 4.923616 \\
\hline 47 & 0.123415 & 41 & 1.709013 & 24 & 5.026785 \\
\hline 31 & 0.135982 & 42 & 2.257031 & 25 & 5.435194 \\
\hline 32 & 0.183106 & 43 & 2.327745 & 26 & 5.602879 \\
\hline 33 & 0.188574 & 44 & 2.343712 & 27 & 5.697106 \\
\hline 34 & 0.233401 & 45 & 2.533104 & 65 & 6.258768 \\
\hline 35 & 0.258396 & 46 & 2.534721 & 64 & 6.811211 \\
\hline 37 & 0.375555 & 59 & 3.728695 & 63 & 7.191548 \\
\hline 6 & 0.380648 & 60 & 3.739175 & 62 & 7.250012 \\
\hline 38 & 0.411558 & 61 & 3.739175 & & \\
\hline 39 & 0.428671 & 60 & 3.739175 & & \\
\hline 40 & 0.431815 & 61 & 3.739175 & & \\
\hline 7 & 0.542986 & 15 & 3.798987 & & \\
\hline 8 & 0.731318 & 16 & 3.938504 & & \\
\hline 51 & 0.811226 & 58 & 4.059528 & & \\
\hline 52 & 0.834372 & 17 & 4.194888 & & \\
\hline 48 & 0.836456 & 18 & 4.198091 & & \\
\hline 9 & 0.849895 & 14 & 4.264913 & & \\
\hline 53 & 0.852150 & 13 & 4.375873 & & \\
\hline 54 & 0.865271 & 19 & 4.408591 & & \\
\hline
\end{tabular}

TABLE VII \% VOLTAGE DEVIATION W.R.T PU VOLTAGE OF 69 NODE RADIAL DISTRIBUTION NETWORK [12] IN ASCENDING ORDER FOR \% DEVIATION LESS THAN 1.000 AFTER NETWORK RECONFIGURATION

\begin{tabular}{|c|c|c|c|}
\hline Node Number & $\%$ Voltage Deviation & Node Number & $\%$ Voltage Deviation \\
\hline 2 & 0.003308 & 38 & 0.254089 \\
\hline 3 & 0.006617 & 39 & 0.290011 \\
\hline 28 & 0.007308 & 40 & 0.292091 \\
\hline 4 & 0.013586 & 7 & 0.318519 \\
\hline 29 & 0.014480 & 8 & 0.351306 \\
\hline 36 & 0.014736 & 51 & 0.354811 \\
\hline 30 & 0.026597 & 52 & 0.355754 \\
\hline 5 & 0.026799 & 48 & 0.361241 \\
\hline 47 & 0.026984 & 9 & 0.363792 \\
\hline 31 & 0.028738 & 53 & 0.372024 \\
\hline 32 & 0.039432 & 54 & 0.380840 \\
\hline 33 & 0.065077 & 55 & 0.386709 \\
\hline 34 & 0.098683 & 56 & 0.386709 \\
\hline 35 & 0.105438 & 10 & 0.497600 \\
\hline 37 & 0.129826 & 11 & 0.524161 \\
\hline 6 & 0.169935 & 66 & 0.529762 \\
\hline
\end{tabular}




\begin{tabular}{|c|c|c|}
\hline Node Number & $\begin{array}{l}\text { Real Load(Pl) } \\
\text { Increase }(\mathrm{kW})\end{array}$ & $\begin{array}{c}\text { Reactive Load(Q1) } \\
\text { Increase(kVAR) }\end{array}$ \\
\hline 2 & 0.0000 & 0.0000 \\
\hline 3 & 0.0000 & 0.0000 \\
\hline 28 & 42.120 & 30.132 \\
\hline 4 & 0.0000 & 0.0000 \\
\hline 29 & 42.120 & 30.132 \\
\hline 36 & 42.120 & 30.051 \\
\hline 30 & 0.0000 & 0.0000 \\
\hline 5 & 0.0000 & 0.0000 \\
\hline 47 & 0.0000 & 0.0000 \\
\hline 31 & 0.0000 & 0.0000 \\
\hline 32 & 0.0000 & 0.0000 \\
\hline 33 & 22.680 & 16.200 \\
\hline 34 & 31.590 & 22.680 \\
\hline 35 & 9.7200 & 6.4800 \\
\hline 37 & 42.120 & 30.051 \\
\hline 6 & 4.2120 & 3.5640 \\
\hline 38 & 0.0000 & 0.0000 \\
\hline 39 & 38.880 & 27.540 \\
\hline 40 & 38.880 & 27.540 \\
\hline 7 & 65.448 & 48.600 \\
\hline 8 & 121.50 & 87.480 \\
\hline 51 & 65.610 & 45.846 \\
\hline 52 & 5.8320 & 4.3740 \\
\hline 48 & 127.98 & 91.368 \\
\hline 9 & 48.600 & 35.640 \\
\hline 53 & 7.0470 & 5.6700 \\
\hline 54 & 42.768 & 30.780 \\
\hline 56 & 0.0000 & 0.0000 \\
\hline 10 & 0.0000 & 0.0000 \\
\hline 11 & 45.360 & 30.780 \\
\hline 66 & 234.90 & 168.48 \\
\hline
\end{tabular}

TABLE IX COMPARISON OF REAL AND REACTIVE POWER LOSSES

\begin{tabular}{|c|c|c|c|}
\hline & Number of Iterations & Real Power Loss (kW) & $\begin{array}{c}\text { Reactive Power } \\
\text { Loss(kVAR) }\end{array}$ \\
\hline Before Network Reconfiguration & 5 & 224.933 & 102.129 \\
\hline After Network Reconfiguration & 4 & 104.939 & 99.059 \\
\hline
\end{tabular}

TABLE X COMPARISON OF MINIMUM PU VOLTAGE LEVEL AND RESPECTIVE NODE NUMBER

\begin{tabular}{|c|c|c|c|}
\hline & $\begin{array}{c}\text { Number of } \\
\text { Iterations }\end{array}$ & $\begin{array}{c}\text { Branch } \\
\text { Number }\end{array}$ & $\begin{array}{c}\text { Voltage } \\
\text { Level }(\mathrm{pu})\end{array}$ \\
\hline $\begin{array}{c}\text { Before Network } \\
\text { Reconfiguration }\end{array}$ & 5 & 65 & 0.909191 \\
\hline $\begin{array}{c}\text { After Network } \\
\text { Reconfiguration }\end{array}$ & 4 & 61 & 0.949483 \\
\cline { 2 - 4 } & 4 & 62 & 0.932401 \\
\hline
\end{tabular}

TABLE XI COMPARISON OF TOTAL REAL AND REACTIVE POWER LOAD

\begin{tabular}{|c|c|c|c|}
\hline & $\begin{array}{c}\text { Number of } \\
\text { Iterations }\end{array}$ & $\begin{array}{c}\text { Real Power } \\
\text { Load (TPL) }\end{array}$ & $\begin{array}{c}\text { Reactive } \\
\text { Power } \\
\text { Load(TQL) }\end{array}$ \\
\hline $\begin{array}{l}\text { Before Network } \\
\text { Reconfiguration }\end{array}$ & 5 & 3801.890090 & 2692.599781 \\
\hline $\begin{array}{l}\text { After Network } \\
\text { Reconfiguration }\end{array}$ & 4 & 3801.890090 & 2693.599649 \\
\cline { 2 - 4 } & 4 & 4226.186872 & 2997.647785 \\
\hline
\end{tabular}




\section{CONCLUSION}

A formula for maximum loading of conductor for radial distribution network is proposed in the paper to compute the change in loading of the radial network. In this proposed method, the most sensitive node and the node having the minimum voltage deviation are found and operated in this paper, nodes having voltage deviation less than $1 \%$ are operated. The critical loadings of the 69-node radial distribution network [12] have been found out for constant power load modelling for substation voltage of 1.0 p.u. and the results are obtained by the proposed method. The comparison of various switching options will show that the critical loading by the proposed method is superior. The overall loss i.e., real and reactive power losses are reduced, voltage profile of the network is improved and total loading of network is increased.

\section{REFERENCES}

[1] H.N Tram and D.L Wall, "Optimal Conductor Selection in Planning Radial Distribution Systems", IEEE Trans. Power Systems, Vol. 3, No. 1, 1988, pp. 200-206.

[2] M. Ponnavaikko and K.S. Prakasa " An Approach to Distribution System Planning Through Conductor Gradation" IEEE Trans. Power Systems, Vol. PAS̃ 101, No. 6, 1982, pp. 1735-1742.

[3] P.S. Nagendra Rao, "An Extremely Simple Method of Determining Optimal Conductor Sections For Radial Distribution Feeders", IEEE Trans. Power Systems, Vol. PAS̃ 104, No.6, 1985, pp.1439-1442.

[4] S. Salamat Sharif, M.M.A Salma, A. Vannelli, " Optimal Model for Future Expansion of Radial Distribution Networks Using Mixed Integer programming", IEEE Trans. Power Systems, Vol.3, No.3, 1988, pp. $970-977$.

[5] Zhuding Wang, Haijun Liu, David C Yu, Xiaohui Wang and Hongquan Song "A Practical Approach to the Conductor Size Selection in Planning Radial Distribution Systems ”, IEEE Trans. Power Systems, Vol.15, No.1, 2000, pp. 350-354.

[6] Karen Nan Miu and Hsiao-Dong Chiang, "Electrical Distribution Load Capability: Problem Formulation, Solution Algorithm, and Numerical Results", IEEE Trans. Power Delivery, Vol.15, No.1, 2000, pp. $43 \tilde{6}$ 442 .

[7] D. Das, "Maximum Loading and Cost of Energy Loss of Radial Distribution Feeders ", International Journal of Electrical Power and Energy Systems, Vol.26, No. 1, 2004, pp. 307-314.

[8] W.G. Kirn and R.B Adler, "A Distribution System Cost model and its Application to Optimal Conductor Sizing", IEEE Trans. Power Apparatus and Systems, Vol.PAS̃ 101, No.2, 1982, pp. 271-275.

[9] S. Satyanarayana, T. Ramana, G.K. Rao and S.Sivanagaraju, "Improving the Maximum Loading of Conductor by Optimal Conductor Selection of Radial Distribution Systems", International Journal of Electrical Power Components and Systems, Vol.34, 2006, pp. 747-757.

[10] S. Ghosh and D. Das, "An Efficient Algorithm for Loss Minimization via Network Reconfiguration," Electric Power Components and Systems, Vol. 31, 2003, pp. 791-804.

[11] S. Ghosh and D. Das, "Method for Load-Flow Solution of Radial Distribution Networks", Proceedings on IEE Part C (GTD), Vol. 146, No. 6,1999 , pp. $641-648$.

[12] M.E. Baran and F.F. Wu, "Optimal Sizing of Capacitors Placed on a Radial Distribution System", IEEE Trans. Power Delivery, Vol. 4, No.1, 1989, pp. $35-743$.

Smarajit Ghosh is presently serving as a Professor and Head, Department of Electrical and Instrumentation Engineering, Thapar University. He did his B.Tech., M.Tech. in Electrical Machines and Power Systems from Calcutta University in 1994 and 1996 respectively. Finally, he did his Ph.D. from Indian Institute of Technology, Khapargpur, India in 2000. His research areas include load-flow study, network reconfigurations, optimum capacitor allocation, application of soft computing in Electrical Power Distribution Systems

Uttamjit Singh Chatwal did his M.Tech from the Department of Electrical and Instrumentation Engineering, Thapar University, Patiala in 2008. At present he is serving in BUILT. His area of interest is in Electric Power Distribution Systems. 